\title{
Fever, Hemiparesis and Hyponatraemia in a 63-year-old. HIV, another Great Masquerader?
}

\author{
David Agius, Jonathan Gauci, Lars Aakeroy, Brendan Caruana Montaldo \\ Department of Medicine, Mater Dei Hospital, Msida, Malta
}

Received: $13 / 05 / 2015$

Accepted: $25 / 06 / 2015$

Published: $27 / 07 / 2015$

How to cite this article: Agius D, Gauci J, Aakeroy L, Caruana Montaldo B. Fever, hemiparesis and hyponatraemia in a 63-year.old. HIV, another Great Masquerader?. EJCRIM 2015;2:doi: 10.12890/2015_000232

Conflicts of Interests: The authors declare that they have no competing interests.

Acknowledgements: The authors declare they have obtained patient's permission.

This article is licensed under a Commons Attribution Non-Commercial 4.0 License

ABSTRACT

The authors describe the unusual case of a 63-year-old patient who was referred with fever and lethargy, and was found to be hyponatraemic. The patient subsequently developed hemiparesis, and neuroradiology showed several space-occupying brain lesions. The cause was later identified as cerebral toxoplasmosis in undiagnosed Acquired Immunodeficiency Syndrome (AIDS).

\section{LEARNING POINTS}

- Early detection and treatment of Acquired Immunodeficiency Syndrome (AIDS) is important in the prevention of associated neurological sequelae.

- Human Immunodeficiency Virus (HIV) testing should be considered in cases of unexplained fever and lethargy even in the absence of risk factors for transmissible diseases.

- AIDS may present as unexplained hyponatraemia due to the syndrome of inappropriate antidiuretic hormone secretion (SIADH).

\section{KEYWORDS}

Toxoplasmosis, HIV, Acquired Immunodeficiency Syndrome, Inappropriate ADH Syndrome.

\section{CASE PRESENTATION}

A 63-year-old man was referred to the Emergency Department with a 4-week history of lethargy, weight loss, fever, and night sweats. He had a fever of $38^{\circ} \mathrm{C}$ at presentation, but there were no localising symptoms or signs of infection. He had a past history of giardiasis, diagnosed on duodenal biopsy 1 year previously, which was treated effectively with metronidazole. A thorough social history was non-contributory.

The patient was admitted for further diagnostic investigation, and initial tests showed mild pancytopenia and an elevated Erythrocyte Sedimentation Rate of $80 \mathrm{~mm} /$ hour. Chest X-ray, blood and urine cultures were normal. He was found to be hyponatraemic with a serum sodium level of $122 \mathrm{mmol} / \mathrm{l}$. Further testing revealed a low serum osmolarity and a high urine osmolarity, in the presence of a normal thyroid and adrenal function, suggesting the presence of the syndrome of inappropriate antidiuretic hormone secretion (SIADH). Two days post admission, the patient was increasingly lethargic and a full neurological examination was repeated, revealing new left-sided hemiparesis. Computed tomography (CT) of the brain showed a smooth, ring enhancing lesion just lateral to the frontal horn of the right lateral ventricle, with extensive vasogenic oedema. Magnetic Resonance Imaging (MRI) of the brain demonstrated multiple ring enhancing lesions, including a $2.7 \mathrm{~cm}$ lesion in the right basal ganglia (Fig. 1), two smaller lesions at the base of the left frontal sulci, as well as small foci of enhancement in the cerebellum. The patient was started on intravenous ceftriaxone and metronidazole by the on-call physician in Neurology, and the Infectious Diseases team recommended adding oral co-trimoxazole. The patient's serum sodium level improved after antibiotic treatment was initiated.

An underlying cause was sought. Echocardiography excluded vegetations, and computed tomography of the trunk showed mesenteric panniculitis, keeping with the previous diagnosis of Giardiasis. Toxoplasma antibodies and Human Immunodeficiency Virus (HIV) testing were both positive. A diagnosis of cerebral toxoplasmosis was made on the basis of a background of acquired immunodeficiency syndrome (AIDS). A repeat sexual history was taken from both the patient and his wife, and this did not reveal any risk factors for retroviral exposure. The patient made a remarkable recovery over a 2-week period of hospitalization, where he received intensive physiotherapy, along with the antibiotic therapy. The patient regained full strength and was discharged home on co-trimoxazole 17 days into the admission.

The patient was follow-up by physicians in Infectious Diseases 1 week later, when highly active antiretroviral therapy (HAART) was started, including efavirenz, lamivudine and zidovudine. He was followed-up at the Outpatients clinic, and he remained clinically well, with a substantial decrease in the size of the ring enhancing lesions on repeated MRI scans (Fig. 2). 


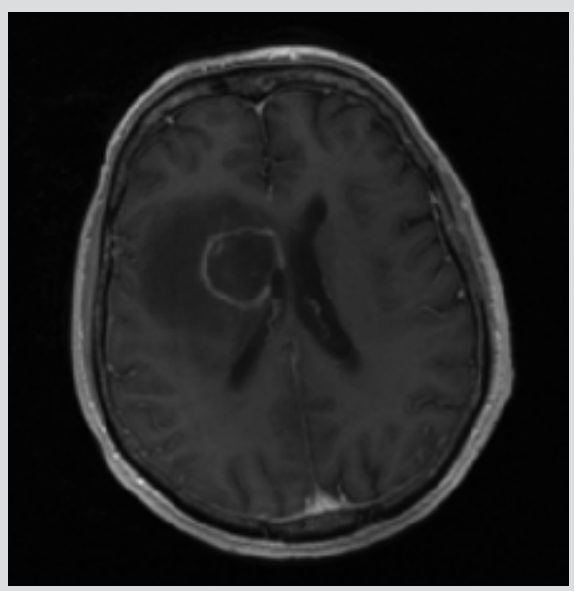

Figure 1: Magnetic resonance imaging (MRI) of the brain, depicting ring enhancing toxoplasma brain abscess

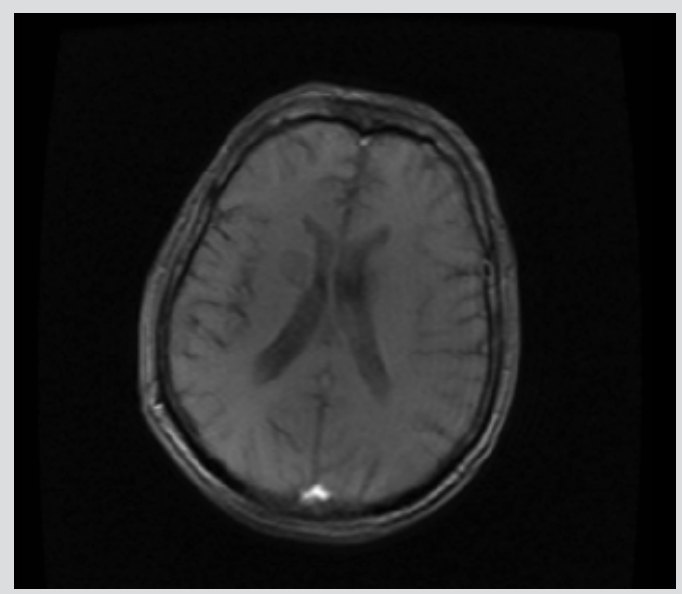

Figure 2: Magnetic resonance imaging (MRI) of the brain, depicting improvement 13 weeks after initiation of co-trimoxazole and 11 weeks after initiation of highly active antiretroviral therapy (HAART)

\section{DISCUSSION}

The authors note that this patient presented 1 year previously with chronic diarrhoea, and was diagnosed with giardiasis on duodenal biopsy. This was probably the actual first presentation of AIDS. Giardia lamblia is a protozoan which can cause diarrhoea and malabsorption in both immunocompetent and immunosuppressed patients. Several studies have revealed a higher prevalence of Giardia lamblia in AIDS patients than in the general population, while other studies have not shown a statistically significant difference ${ }^{[1,2]}$. The treatment of choice is metronidazole, though relapse may occur in AIDS. In retrospect, it is possible that the cerebral toxoplasmosis with its neurological sequelae may have been prevented if the patient had been tested for HIV when he was diagnosed with giardiasis. However, the social history of the patient did not suggest any risk for HIV exposure, and testing all giardiasis patients for HIV is debatable.

The presentation of fever and weight loss without any localising features should raise the suspicion of AIDS. The authors argue that HIV testing may be warranted in such cases, even in the absence of risk factors for transmissible diseases on repeated history-taking. The development of neurological features in the febrile patient further increases the possibility of HIV-related illness. AIDS presents with neurological manifestations in $10-20 \%$ of cases, and toxoplasma gondii has long been recognised as the most common cause for the neurological sequelae ${ }^{[3]}$. These vary from focal deficits to an encephalitic picture with headache, confusion and seizures. Hemiparesis, aphasia, ataxia, and diplopia are the most common focal neurological deficits ${ }^{[4]}$.

The diagnosis of cerebral toxoplasmosis is supported by positive serology and neuroimaging with CT or MRI. Toxoplasma seropositivity rates in HIV patients varies in different populations from 10 to $50 \%$, with the highest incidence occurring in Africa ${ }^{[5]}$. Toxoplasma immunoglobulin $\mathrm{G}(\operatorname{lgG})$ antibodies have a high sensitivity, with $97 \%$ of patients with cerebral toxoplasmosis having a positive result ${ }^{[6]}$. Neuroradiological features vary, but it has been shown that $60 \%$ to $70 \%$ of patients have bilateral, multiple, hypodense ring-enhancing lesions, with surrounding edema on $\mathrm{CT}^{[7]}$. MRI is more sensitive and may detect additional lesions. The differential diagnosis of multiple-enhancing mass lesions includes abscesses (bacterial, tuberculous, fungal and toxoplasma), metastases, lymphoma, gliobastoma multiforme, demyelinating disease, radiation necrosis, subacute infarct, resolving haematoma, and contusion. Positive toxoplasma serology with compatible neuroradiological features in AIDS patients should be treated empirically, with a brain biopsy reserved for patients with negative toxoplasma serology and who do not respond to treatment ${ }^{[8]}$.

The combination of pyrimethamine and sulfadiazine is the gold standard treatment for toxoplasmosis. The combination of trimethoprim and sulfamethoxazole (co-trimoxazole) is also highly effective, and a Cochrane review from 2006 did not show any regimen to be superior ${ }^{[9]}$. The choice of therapy is often directed by availability, and co-trimoxazole is an effective alternative when pyrimethamine/sulfadiazine is not available. Steroids are often prescribed with the aim of reducing cerebral oedema, but it has not been shown that they improve outcome ${ }^{[10]}$. The SIADH is a less common but recognised presentation of AIDS. SIADH is diagnosed in the context of an euvolaemic hyponatraemia with serum hypo-osmolarity and urine hyperosmolarity with the exclusion of severe hypothyroidism and glucocorticoid insufficiency. The likely cause of SIADH was cerebral toxoplasmosis in this patient. Other recognised causes in AIDS patients are cryptococcal meningoencephalitis, pneumonia, pulmonary tuberculosis, and malignancy. The authors note that unexplained hyponatraemia with a finding of SIADH should alert for possible HIV infection.

\section{REFERENCES}

1. Moolasart, P., Giardia lamblia in AIDS patients with diarrhea. J Med Assoc Thai. 1999;82:654-9.

2. Meamar AR, Rezaian M, Mohraz M, Zahabiun F, Hadighi R, Kia EB. A Comparative Analysis of Intestinal Parasitic Infections between HIV+/AIDS Patients and Non-HIV Infected Individuals. Iranian J Parasitol;2007;2:1-6

Berger J R, Moskowitz L, Fischl M, Kelley R E. Neurologic disease as the presenting manifestation of acquired immunodeficiency syndrome. South Med J 1987;80:683-6.

4. Ho Y, Sun H, Chen M, Hsieh S, Sheng W, Chang S. Clinical presentation and outcome of toxoplasmic encephalitis in patients with human immunodeficiency virus type 1 infection. J Microbiol Immunol Infect 2008;41:386-392

5. Osunkalu V, Akanmu SA, Ofomah NJ, Onyiaorah IV, Adediran AA, Akinde RO, Onwuezobe IA. Seroprevalence of Toxoplasma gondii IgG antibody in HIV-infected patients at the Lagos University Teaching Hospital. HIV/AIDS (Auckland, N.Z.) 2011:3:101-5.

6. Skiest DJ, Erdman W, Chang WE, Oz OK, Ware A, Fleckenstein J. SPECT thallium-201 combined with Toxoplasma serology for the presumptive diagnosis of focal central nervous system mass lesions in patients with AIDS. J Infect 2000;40:274-81. 
Porter SB, Sande MA. Toxoplasmosis of the central nervous system in the acquired immunodeficiency syndrome. NEJM 1992;327:1643-8.

Mathews C, Barba D, Fullerton SC. Early biopsy versus empiric treatment with delayed biopsy of non-responders in suspected HIV-associated cerebral toxoplasmosis: a decision analysis. AIDS 1995;9:12431250. 10.1097/00002030-199511000-00005

9. Dedicoat M, Livesley N. Management of toxoplasmic encephalitis in HIV-infected adults (with an emphasis on resource-poor settings). Cochrane Database Syst Rev 2006;19:CD005420.

10. Sonneville R, Schmidt M, Messika J, Ait Hssain A, da Silva D, et al. Neurologic outcomes and adjunctive steroids in HIV patients with severe cerebral toxoplasmosis. Neurology 2012;79:1762-6. 